\title{
Effects of a Four-Week Small-Sided Game and Repeated Sprint Ability Training during and after Ramadan on Aerobic and Anaerobic Capacities in Senior Basketball Players
}

\author{
${ }^{1,2}$ Seifeddine Brini* ${ }^{*}$ Hamza Marzouki, ${ }^{3,4}$ Carlo Castagna, ${ }^{1}$ Anissa Bouassida
}

${ }^{1}$ Research Unit, Sportive Performance and Physical Rehabilitation, High Institute of Sports and Physical Education of Kef, University of Jendouba, Kef, Tunisia. ${ }^{2}$ Faculty of Sciences of Bizerte, University of Carthage, 7021 Zarzouna, Bizerte, Tunisia. ${ }^{3}$ University of Rome Tor Vergata, Rome, Italy. ${ }^{4}$ Fitness Training and Biomechanics Laboratory, Italian Football Federation (FIGC), Technical Department, Coverciano (Florence), Italy.

\begin{abstract}
Background. Most studies related to the effect of Ramadan fasting on aerobic and anaerobic performances found in the literature were concerned with individual sports; even studies on team sports were mostly dedicated to football, and none of those studies were involved basketball competitions. Objectives. This field investigation examined the effects of a training program in the basics of small-sided games (SSGs) and repeated sprint ability (RSA) during Ramadan observance (R) and a month after Ramadan (AR) (control month)on aerobic and ("anaerobic") performances in fasting basketball players. Methods. Sixteen basketball players (age, $23.4 \pm 2.3$ years; height, $1.86 \pm .09 \mathrm{~m}$; body mass, $78.3 \pm 11.0 \mathrm{~kg}$; and $\mathrm{VO}_{2 \max }, 51.0 \pm 2.7 \mathrm{ml} \cdot \mathrm{min}^{-1} \cdot \mathrm{kg}^{-1}$ ) performed two training programs (SSG and RSA) during R and AR, interrupted by fifteen days of total recovery, and were randomly allocated into two groups $\left(\mathrm{G}_{\mathrm{SSG}}, \mathrm{n}=8\right.$ and $\left.\mathrm{G}_{\mathrm{RSA}}, \mathrm{n}=8\right)$. RSA parameters, mean heart rate (HR), and aerobic performances were measured on four occasions: before (P1) and after (P2) R and before (P'1) and after (P'2) AR. Results. The results have shown that RSA and Ramadan show no significant differences in aerobic and anaerobic performances when compared with SSG and non-Ramadan in senior basketball players. However,

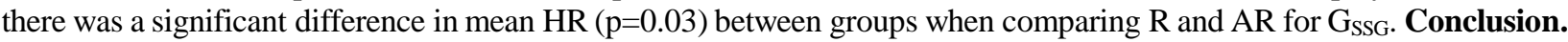
This study indicated that Ramadan fasting combined with SSG and RSA training has no significant effect on most aerobic and anaerobic performances in male senior basketball players.
\end{abstract}

\section{KEY WORDS: Ramadan, Training, Heart Rates, Basketball, Aerobic, Performance, Fatigue.}

\section{INTRODUCTION}

Basketball is an intermittent high-intensity activity that heavily stresses the aerobic and anaerobic metabolism (1). The ability to repeat maximal sprint with short incomplete recovery time (repeated sprint ability [RSA]) was considered a relevant component of basketball performance (2). Furthermore, the aerobic fitness is more important than previously supposed for the determination of basketball match activities. The implementation of basketball-specific drills in the form of smallsided games (SSGs) was suggested as a viable strategy to functionally improve aerobic fitness (3).

\footnotetext{
*. Corresponding Author:

Seifeddine Brini

E-mail: bseifeddine15@gmail.com
} 
Moreover, basketball players continue to train and play decisive games at a high level under extreme climatic conditions and with a sporting calendar that is not modified for religious observances (4).

In this context, we can consider the month of Ramadan as among the extreme conditions that influence the performance of Muslim athletes. During this blessed month, Muslim athletes abstain from all food, drink, and other physical needs during the daylight hours. Furthermore, in this holy month, there are chronological, physiological, and hormonal changes that affected the physical performance, making Ramadan a particular model of prolonged intermittent fasting $(5,6,7,8,9$, and 10).

Previous investigations have examined the effect of Ramadan fasting and physical activity on aerobic and anaerobic performances. Several studies have investigated the effect of Ramadan fasting on anaerobic performance (11), showing that performances in RSA tests were reduced as result of Ramadan observance. (8) examined the $5-$, 10-, and 30-m sprint performance of young national-level judo athletes, reporting no changes in sprint performance during $\mathrm{R}$.

Sprint performance in 10 and $30 \mathrm{~m}$ sprints showed no changes during the second and fourth weeks of Ramadan in 18-year-old junior soccer players (4). Moreover, in the study by (12), observant subjects showed a slightly greater deceleration in the seven sprints (a deterioration of $9.0 \%$ at 2 weeks and $9.3 \%$ at 4 weeks, rather than $7.7 \%$ before Ramadan). This effect was more marked in morning than afternoon tests.

Concerning the effect of Ramadan observance on aerobic performance, (13) measured maximal oxygen intake directly, using a Bruce treadmill protocol. They observed no difference in peak oxygen transport between before Ramadan and after2 and4 weeks of fasting. Moreover, (8) reported no significant changes in Léger test predictions of maximal oxygen intake during $\mathrm{R}$ in their study of elite judo athletes. Likewise, other investigators revealed no changes in the performance of submaximal aerobic exercise during R (14). However, (15) noted some decrease in maximal oxygen intake during the first week of Ramadan, but the subject values also had recovered to pre-fast levels by the fourth week of Ramadan.
To the best of our knowledge, most studies related to the effect of Ramadan fasting on aerobic and anaerobic performances found in the literature were concerned with individual sports (8); even studies on team sports were mostly dedicated to football $(11,12,16)$, and none of those studies were involved basketball competitions, which can cross the month of Ramadan, making our study the first to investigate intermittent fasting effects coupled with athletic training program in basketball. So our assumption was that senior basketball players can intercalate RSA and SSG-based training programs during the month of Ramadan without falling into the trap of overtraining.

In this context, our investigation will be conducted according to two main factors, that is training method (SSG vs. RSA) and Ramadan (Ramadan vs. non-Ramadan [control month]).Therefore, the purpose of the present study was to compare the effects of four-week SSG and RSA training during and after Ramadan on aerobic and anaerobic capacities in senior basketball players.

\section{MATERIALS AND METHODS}

Participants. Sixteen male basketball players (mean \pm SD, age, 23.4 \pm 2.3 years; height, $1.86 \pm 0.09 \mathrm{~m}$; body mass, $78.3 \pm 11.0 \mathrm{~kg}$; body mass index [BMI], 22.60 $\pm 1.95 \mathrm{~kg} \cdot \mathrm{m}^{-2}$; and VO2max, 51.0 $\left.2.7 \mathrm{ml} \cdot \mathrm{min}^{-1} \cdot \mathrm{kg}^{-1}\right)$ from the second division (4-5 days/week, $>60 \mathrm{~min} /$ day, with a training experience of $11.8 \pm 3.9$ years) volunteered for this study. The study was conducted 2 weeks after the end of their competitive season. The subjects were randomly assigned to two groups as follows: a SSG group $\left(\mathrm{G}_{\mathrm{SSG}} ; \mathrm{n}=8\right)$ and RSA group $\left(\mathrm{G}_{\mathrm{RSA}} ; \mathrm{n}=8\right)$. The inclusion criteria for study participation were as follows: (i) participation in at least $90 \%$ of the training sessions, (ii) Muslims who were fasting during Ramadan (R) and (iii) good health (no pain or injury reported) and not receiving any medication or other drugs. The study was approved by a local research ethics committee, and the protocol was conducted according to the Declaration of Helsinki. All participants provided written informed consent to participate in the study.

Design and Procedures. This study aimed to examine a possible Ramadan observation effect 
on players undergoing RSA and SSG intervention. Players were randomly assigned into two training groups, as follows: $\mathrm{G}_{\mathrm{SSG}}(\mathrm{n}=8)$ and $\mathrm{G}_{\mathrm{RSA}}(\mathrm{n}=8)$. The training groups (i.e., $\mathrm{G}_{\mathrm{SSG}}$ and $\mathrm{G}_{\mathrm{RSA}}$ ) performed a four-week training program during $\mathrm{R}$ and a month after Ramadan (AR), interrupted by 15 days of total recovery, with a frequency of two sessions per week. However, on other days of the week, both groups maintained their normal training routine.

During the Ramadan phase of the study, subjects refrained from eating and drinking from dawn to sunset. Players were informed to maintain a similar nutrition and hydration routine while consuming the usual Iftar and Sahur meals close to every test.

The Iftar meal contained a salad, soup, spaghetti, chicken thigh, juice, and an apple, whereas the Sahur meal contained a salad, one yogurt, a piece of cake, and a banana. The same meals were served during the control month. The period of fasting in 2016 was from 03:11 to $19: 36 \mathrm{~h}$ at the beginning of Ramadan to $03: 16$ to $19: 42 \mathrm{~h}$ at the end of Ramadan (Figure1).

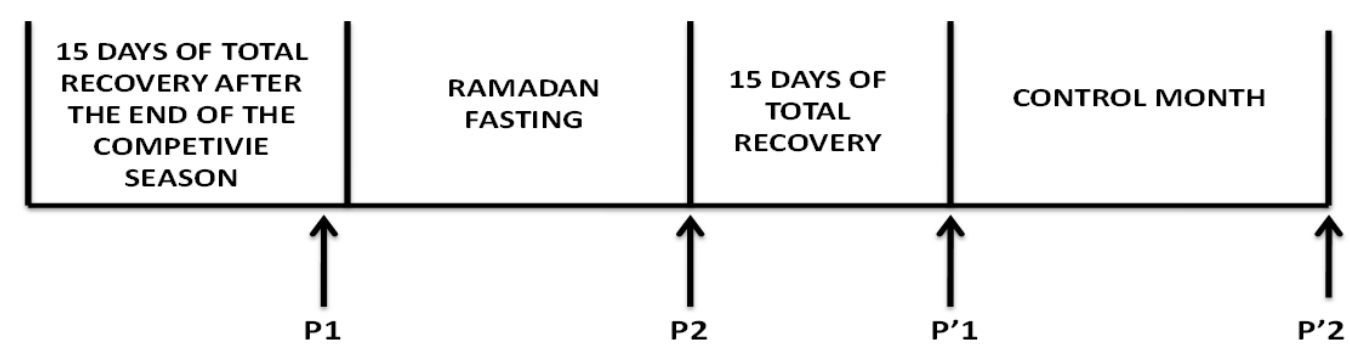

Figure 1. Representation of the experimental protocol. P: Tests (RSA+ yo-yo). P1: before Ramadan; P2: the end of Ramadan; P'1: before control month; P'2: the end of control month.

Training program. Players were part of the same basketball team and performed two training sessions per week with SSG and RSA protocols according to their group allocation $\left(\mathrm{G}_{\mathrm{SSG}}\right.$ and $\mathrm{G}_{\mathrm{RSA}}$ ) at least $24 \mathrm{~h}$ apart. The session started with a 15-min standardized warm-up (5 min of lowintensity running, $5 \mathrm{~min}$ of dynamic stretching, and $5 \mathrm{~min}$ of skipping) followed by the experimental training. The remaining training time was dedicated to specific basketball training.

The SSG drill used was in the form of $2 \mathrm{v} 2$ on full-length $(28 \mathrm{~m})$ and half-width $(7.5 \mathrm{~m})$
court.The $2 \mathrm{v} 2$ format was chosen because it was deemed to induce a greater intensity than other SSG drills involving more players $(17,18)$. (Table 1) Drills were played like a competition, with only man-to-man defense, and no free throws or time-outs. Scores were kept to encourage players' motivation and verbal encouragements were provided by the coach. Players were then randomly allocated to pairs (composed of a guard and either a forward or a centre), and new pairs were created for each training session.

Table 1. Description of the 4-weeks training program for the small-sided game (SSG) group and the repeated sprint ability group (RSA)

\begin{tabular}{ccc}
\hline & SSG & RSA \\
\hline Week 1 & $2 \times(2 \times 3 \min 45)$ & $3 \times 6 \times 30 \mathrm{~m}(15+15) 20 \mathrm{~s} \mathrm{rec}$ \\
Week 2 & $2 \times(2 \times 3 \min 45)$ & $3 \times 6 \times 30 \mathrm{~m}(15+15) 20 \mathrm{~s} \mathrm{rec}$ \\
Week 3 & $2 \times(3 \times 4 \min 30)$ & $3 \times 8 \times 30 \mathrm{~m}(15+15) 20 \mathrm{~s} \mathrm{rec}$ \\
Week 4 & $2 \times(3 \times 4 \min 30)$ & $3 \times 8 \times 30 \mathrm{~m}(15+15) 20 \mathrm{~s} \mathrm{rec}$ \\
\hline
\end{tabular}

The RSA consisted of three sets of 30-m maximal shuttle running $(15 \mathrm{~m})$ sprints, after every 20 s of passive recovery,repeated 6 and 8 times in the first two and last two weeks of the intervention, respectively (19). The between-set recovery was 4 min (Table 1). 
Session rating of perceived exertion (session-RPE). The session-RPE method was used to determine the training program load, 30 min after the end of the training session for both groups (Borg's CR-10 scale) (21). Players were largely familiar with this method, which was regularly used during their training program.

Heart rate (HR) measurements. The HR during RSA tests was recorded continuously using a cardiofrequency monitor (Polar Electric, Kempten, Germany).

Anthropometrics measurements. Weight $(\mathrm{kg})$ was measured by an electronic balance (Pharo 200) and height (m) was measured with a stadiometer ( Seca 217 ). BMI was calculated as follows: BMI $\left(\mathrm{kg} / \mathrm{m}^{2}\right)=$ weight $/$ height $^{2}$.

Yo-Yo intermittent recovery test. The YoYo test was performed according to the procedures suggested by (22). The test consists of $20-\mathrm{m}$ shuttle runs performed at increasing velocities with $10 \mathrm{~s}$ of active recovery between runs until exhaustion. Audio cues of the Yo-Yo test were recorded on a CD (Teknosport.com, Ancona, Italy) and broadcasted using a portable calibrated CD player (Philips, Az1030 CD player, Eindhoven, Holland). The test is considered finished when the participant fails to reach the front line in time twice or feels unable to complete another shuttle at the dictated speed. The total distance (TD) covered during the Yo-Yo test level 1 (including the last incomplete shuttle) is calculated and stored for further analyses.

RSA test. The RSA test (17) consisted of ten $30-\mathrm{m}$ shuttle sprints $(15 \mathrm{~m}+15 \mathrm{~m})$ each with a single change in direction of $180^{\circ}$, intercepted by $30 \mathrm{~s}$ of passive recovery. The participants sprinted linearly from the start line to $15 \mathrm{~m}$, touched a line on the floor with a foot, and then, following a $180^{\circ}$ change in direction, returned to the start line as fast as possible. The times of different repetitions are recorded with the aid of the photocells (Brower Timing System, Salt Lake City, UT, USA; accuracy of $0.01 \mathrm{~s}$ ).

Statistical analysis. Data analyses were performed using SPSS version 17 for Windows (SPSS Inc., Chicago, IL, USA). Values are presented as mean \pm SD. The normality of data sets was checked using the Kolmogorov-Smirnov test. Compound symmetry was analyzed using the Mauchlin test. Two-way analysis of variance (ANOVA) with repeated measures was used to determine the differences between groups (twocondition group, $\mathrm{G}_{\mathrm{SSG}}$ or $\mathrm{G}_{\mathrm{RSA}} \times$ time of measurement, P1, P2).When a difference was found, a Bonferroni post hoc test was performed. We calculated effect sizes (ES) for each output (23) and 95\% confidence interval (ESCI) when significant between-time or between-group differences were found. Paired t-test was applied to compare the aerobic and anaerobic changes observed after 4 weeks of training during $\mathrm{R}$ compared to those recorded during AR. Statistical significance was assigned at $\mathrm{P}$-value $<0.05$ for all analyses.

\section{RESULTS}

Data in Table 2 present the effects of Ramadan fasting combined with two training programs (SGG and RSA) and AR on RSA and aerobic performances in basketball players.

The results showed that there was no significant effect after four weeks of Ramadan intermittent fasting on most RSA parameters, HR, and aerobic performance for both groups.

Data in Table 3 show the delta variation of RSA parameters and TD performance measured in subjects during $\mathrm{R}$ and $\mathrm{AR}$.

The delta variation of the best time (TPIC) indicates a significant difference by comparing Ramadan with the control month for both $\mathrm{G}_{S S G}(\mathrm{R}$ [0.07] vs. AR [0.02], 0.02 [S] ) and $\mathrm{G}_{\mathrm{RSA}}(\mathrm{R}$ [0.07] vs. AR [0.01], 0.05 [S]).

Concerning the delta variation of mean HR measured during the RSA test, there were significant differences in the $\mathrm{G}_{S S G}$ by comparing Ramadan with the control month (R [-1.54 ]vs. AR [0.3], 0.0393[S]); however, there were no significant differences in the $\mathrm{G}_{\mathrm{RSA}}(\mathrm{R}$ vs. $\mathrm{AR}$, $0.1038[\mathrm{NS}])$.

Statistical analysis revealed that there were no significant differences concerning RPE scores when comparing Ramadan with the control month (AR) for both groups.

\section{DISCUSSION}

According to the two main factors in our investigation, which are the training method (SSG vs. RSA) and Ramadan (Ramadan vs. nonRamadan [control month]), the findings have shown that RSA and Ramadan have no significant differences in aerobic and anaerobic performances when compared with the SSG and non-Ramadan in senior basketball players. 
Table 2. Data expressed as mean \pm SD of RSA parameters, heart rate mean and TD performance measured in subjects during Ramadan and month after Ramadan.

\begin{tabular}{ccccc|ccc}
\hline & & \multicolumn{2}{c}{ The Fasting month of Ramadan } & \multicolumn{3}{c}{ The Month after Ramadan } \\
\cline { 3 - 7 } & & P1 & P2 & ES & P'1 & P'2 & ES \\
\hline \multirow{2}{*}{ TT(s) } & $\mathrm{G}_{\text {SSG }}$ & $59,78 \pm 0,68$ & $58,42 \pm 2,44$ & 0,80 & $59,84 \pm 0,62$ & $59,58 \pm 0,70$ & 0,04 \\
& $\mathrm{G}_{\mathrm{RSA}}$ & $59,72 \pm 1,14$ & $59,22 \pm 1,32$ & 0,11 & $59,81 \pm 1,11$ & $59,65 \pm 1,08$ & 0,02 \\
\hline \multirow{2}{*}{ TPIC(s) } & $\mathrm{G}_{\text {SSG }}$ & $5,90 \pm 0,11$ & $5,83 \pm 0,14$ & 0,02 & $5,91 \pm 0,09$ & $5,89 \pm 0,10$ & 0,007 \\
& $\mathrm{G}_{\mathrm{RSA}}$ & $5,88 \pm 0,13$ & $5,81 \pm 0,17$ & 0,02 & $5,90 \pm 0,12$ & $5,89 \pm 0,11$ & 0,008 \\
\hline \multirow{2}{*}{ FI\% } & $\mathrm{G}_{\text {SSG }}$ & $1,32 \pm 0,75$ & $0,17 \pm 3,45$ & 1,17 & $1,30 \pm 0,55$ & $1,12 \pm 0,53$ & 0,10 \\
& $\mathrm{G}_{\mathrm{RSA}}$ & $1,61 \pm 0,57$ & $1,96 \pm 1,02$ & 0,32 & $1,40 \pm 0,45$ & $1,34 \pm 0,36$ & 0,12 \\
\hline \multirow{2}{*}{ HRmean (beat/min) } & $\mathrm{G}_{\text {SSG }}$ & $183,83 \pm 4,77$ & $185,36 \pm 4,50$ & 0,26 & $186,83 \pm 4,46$ & $186,53 \pm 2,75$ & 0,80 \\
& $\mathrm{G}_{\mathrm{RSA}}$ & $186,90 \pm 5,75$ & $188,55 \pm 4,17$ & 0,73 & $189,03 \pm 2,95$ & $189,33 \pm 2,74$ & 0,30 \\
\hline \multirow{2}{*}{ TD(m) } & $\mathrm{G}_{\mathrm{SSG}}$ & $1792,50 \pm 208,65$ & $2065,00 \pm 331,19$ & 0,70 & $1837,50 \pm 181,88$ & $2022,50 \pm 291,68$ & 0,55 \\
& $\mathrm{G}_{\mathrm{RSA}}$ & $1627,50 \pm 412,79$ & $1805,00 \pm 529,66$ & 0,52 & $1667,50 \pm 441,68$ & $1852,50 \pm 499,48$ & 0,45 \\
\hline
\end{tabular}

Data are expressed as mean \pm SD; $\mathbf{G}_{\text {ssG }}$ : small-sided games group; $\mathbf{G}_{\mathrm{RSA}}$ : repeated sprint ability; TT: total time; TPIC: best time group; FI: fatigue index; HR: heart rate; TD: total distance covered in YOYO test; P1: before Ramadan; P2: during the ${ }^{\text {th }}$ week of Ramadan; P'1: before AR ; P'2: during the $4^{\text {th }}$ week of AR; ES: effect size; *: $p<0.05 ; * *$ : $<0.005$.

Table 3. Data expressed as mean \pm SD expressing delta variation of RSA parameters and TD performance measured in subjects during Ramadan and month after Ramadan.

\begin{tabular}{|c|c|c|c|}
\hline & & The Fasting month of Ramadan & The Month after Ramadan \\
\hline & & $\Delta$ P2-P1 & $\Delta \mathrm{P}^{\prime} \mathbf{2}-\mathrm{P}^{\prime}{ }_{1}$ \\
\hline \multirow{2}{*}{ TT(s) } & GSSG $_{\text {S }}$ & $-1,36 \pm 2,24$ & $-0,25 \pm 0,10$ \\
\hline & $\mathrm{G}_{\mathrm{RSA}}$ & $-0,50 \pm 0,32$ & $-0,16 \pm 0,06^{*}$ \\
\hline \multirow[b]{2}{*}{ TPIC(s) } & $\mathrm{G}_{S S G}$ & $-0,07 \pm 0,06$ & $-0,01 \pm 0,02$ \\
\hline & $\mathrm{G}_{\mathrm{RSA}}$ & $-0,07 \pm 0,06$ & $-0,01 \pm 0,02$ \\
\hline \multirow{2}{*}{ FI\% } & $\mathrm{G}_{\mathrm{SSG}}$ & $-1,15 \pm 3,30$ & $-0,17 \pm 0,28$ \\
\hline & $\mathrm{G}_{\mathrm{RSA}}$ & $0,34 \pm 0,92$ & $-0,06 \pm 0,33$ \\
\hline \multirow{2}{*}{$\begin{array}{c}\text { HR } \\
\text { mean(beat/min) }\end{array}$} & $\mathrm{G}_{\mathrm{SSG}}$ & $1,54 \pm 0,73$ & $-0,3 \pm 2,23^{*}$ \\
\hline & GRSA & $1,65 \pm 2,08$ & $0,30 \pm 0,84$ \\
\hline \multirow{2}{*}{ TD(m) } & $\mathrm{G}_{\mathrm{SSG}}$ & $272,5 \pm 196,81$ & $185 \pm 155,56$ \\
\hline & $\mathrm{G}_{\mathrm{RSA}}$ & $177,5 \pm 147,53$ & $185 \pm 128,62$ \\
\hline
\end{tabular}

Data are expressed as mean \pm SD; GssG: small-sided games group; GRSA: repeated sprint ability; TT: total time; TPIC: best time group; FI: fatigue index; HR: heart rate; TD: total distance covered in YOYO test; P1: before Ramadan; P2: during the $4^{\text {th }}$ week of Ramadan; P'1: before AR ; P'2: during the $4^{\text {th }}$ week of AR; ES: effect size; $*: \mathrm{p}<0.05 ; * *: \mathrm{p}<0.005$

Regarding aerobic performances, the TD covered during the Yo-Yo intermittent recovery test did not differ between the two sessions of measurement and between groups by comparing $\mathrm{R}$ and $\mathrm{AR}$, which is in the same line with the study conducted by (8) that showed that estimated values for maximal aerobic velocity and VO2max during the multistage fitness test were relatively unchanged during $\mathrm{R}$ in elite judo athletes.

However, (24) reported that fasting resulted in a significant reduction in aerobic capacity in the 3000-m run. (12) and (11) recently found a significant reduction in the performance in the Yo-Yo intermittent recovery test during $\mathrm{R}$ in young football players, and this significant reduction of performances during $\mathrm{R}$ can be explained when fasting is associated with catecholamine inhibition and reduced venous return, causing a decrease in the sympathetic tone, which leads to a decrease in blood pressure, HR, and cardiac output $(25,26)$.

Concerning anaerobic performances, the present study showed that the total time (TT), TPIC, and fatigue index (FI) during the RSA test did not differ significantly at the end of Ramadan. Furthermore, there were no significant differences between both groups.

Besides, our study disagrees with that of (22), which showed a decrease in speed endurance measured by $4 \times 10-\mathrm{m}$ run time (i.e., an increased sum of the six sprint times and performance decrement during the RSA test). These authors showed a significant mean performance decrement of $9.5 \%$ at the end of Ramadan 
compared to $9.0 \%$ before Ramadan. In addition, (22) showed that the sum of the six sprint times increased from $46.36 \mathrm{~s}$ before the beginning of the Ramadan to $46.73 \mathrm{~s}$ at the end of the month. Moreover, in agreement with previous findings, (11) showed a significant decrease in the highest power output during a $5 \times 6$-s RSA test during R. These changes may reflect a decreased glycolytic capacity and slower replenishment of muscle creatine phosphate stores during the short recovery period between the sprints during $R$.

Otherwise, the mean HR measured during the RSA test did not differ significantly by comparing P1 and P2 between groups. In fact, our study is the first to examine the HR variation in the RSA test during $\mathrm{R}$, so there are no other studies to compare with; however, it seems that several previous studies (14, 27, and 16) reviled a significant decrement in $\mathrm{HR}$ during $\mathrm{R}$ after endurance exercises, as explained by changes in blood pressure and cardiac output.

The present study has some limitations. First, the intervention program was of short duration. Second, the relatively small number of participants may have underpowered the study. Also, we did not include the factor of the competitive season (season vs. recovery) what will be the goal of our future research in relation to this investigation. Finally, we did not use a control group, which was substituted by a control month.

\section{CONCLUSION}

This study indicated that Ramadan observance combined with RSA and SSG has no significant effect on the aerobic and anaerobic performances of both groups. However, the mean HR was higher for GSSG during $\mathrm{R}$ than in the control month (AR).

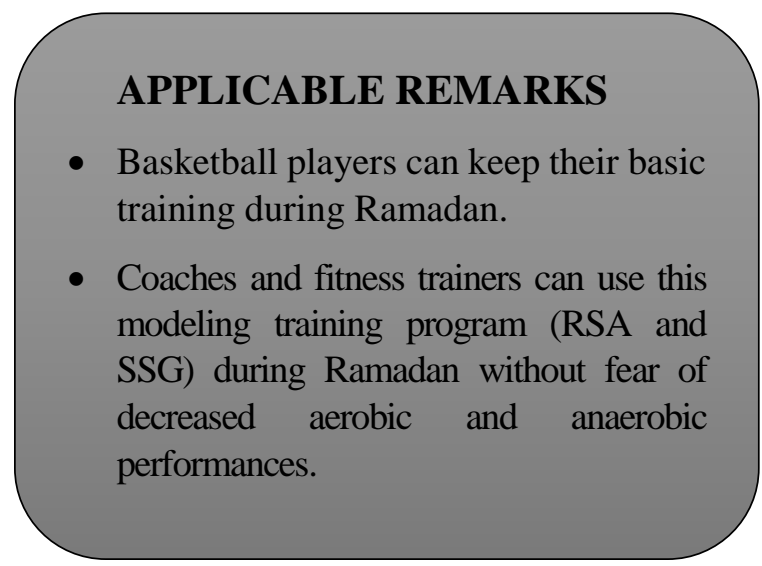

\section{ACKNOWLEDGMENTS}

The authors would like to acknowledge with considerable gratitude all those who volunteered to take part in this study.

\section{REFERENCES}

1. Ben Abdelkrim N, El Fazaa S, El Ati J. Time-motion analysis and physiological data of elite under-19-year-old basketball players during competition. British journal of sports medicine. 2007;41(2):69-75.

2. Castagna C, Abt G, Manzi V, Annino G, Padua E, D'Ottavio S. Effect of recovery mode on repeated sprint ability in young basketball players. Journal of strength and conditioning research. 2008;22(3):923-9.

3. Delextrat A, Martinez A. Small-sided game training improves aerobic capacity and technical skills in basketball players. International journal of sports medicine. 2014;35(5):385-91.

4. Kirkendall DT, Leiper JB, Bartagi Z, Dvorak J, Zerguini Y. The influence of Ramadan on physical performance measures in young Muslim footballers. Journal of sports sciences. 2008;26 Suppl 3:S15-27.

5. Leiper JB, Molla AM, Molla AM. Effects on health of fluid restriction during fasting in Ramadan. European journal of clinical nutrition. 2003;57 Suppl 2:S30-8.

6. Roky R, Houti I, Moussamih S, Qotbi S, Aadil N. Physiological and chronobiological changes during Ramadan intermittent fasting. Annals of nutrition \& metabolism. 2004;48(4):296-303.

7. Souissi N, Souissi M, Souissi H, Chamari K, Tabka Z, Dogui M, et al. Effect of time of day and partial sleep deprivation on short-term, high-power output. Chronobiology international. 2008;25(6):1062-76.

8. Chaouachi A, Coutts AJ, Chamari K, Wong del P, Chaouachi M, Chtara M, et al. Effect of Ramadan intermittent fasting on aerobic and anaerobic performance and perception of fatigue in male elite judo athletes. Journal of strength and conditioning research. 2009;23(9):2702-9.

9. Mujika I, Chaouachi A, Chamari K. Precompetition taper and nutritional strategies: special reference to training during Ramadan intermittent fast. British journal of sports medicine. 2010;44(7):495-501..

10. Waterhouse J. Effects of Ramadan on physical performance: chronobiological considerations. British journal of sports medicine. 2010;44(7):509-15.. 
11. Hamouda O, Chtourou H, Farjallah MA, Davenne D, Souissi N. The effect of Ramadan fasting on the diurnal variations in aerobic and anaerobic performances in Tunisian youth soccer players. Biological Rhythm Research. 2012;43(2):177-90.

12. Chtourou H, Hammouda O, Souissi H, Chamari K, Chaouachi A, Souissi N. The Effect of Ramadan Fasting on Physical Performances, Mood State and Perceived Exertion in Young Footballers. Asian Journal of Sports Medicine. 2011;2(3):177-85.

13. Ramadan J. Does Fasting during Ramadan Alter Body Composition, Blood Constituents and Physical Performance? Medical Principles and Practice. 2002;11 (Suppl. 2):41-6.

14. Ramadan JM, Barac-Nieto M. Cardio-respiratory responses to moderately heavy aerobic exercise during the Ramadan fasts. Saudi medical journal. 2000;21(3):238-44.

15. Sweileh N, Schnitzler A, Hunter GR, Davis B. Body composition and energy metabolism in resting and exercising muslims during Ramadan fast. The Journal of sports medicine and physical fitness. 1992;32(2):156-63.

16. Zerguini Y, Kirkendall D, Junge A, Dvorak J. Impact of Ramadan on physical performance in professional soccer players. British journal of sports medicine. 2007;41(6):398-400.

17. Castagna C, Manzi V, D'Ottavio S, Annino G, Padua E, Bishop D. Relation between maximal aerobic power and the ability to repeat sprints in young basketball players. Journal of strength and conditioning research. 2007;21(4):1172-6.

18. Delextrat A, Kraiem S. Heart-Rate Responses by Playing Position During Ball Drills in Basketball. International Journal of Sports Physiology and Performance. 2013;8(4):410-8.

19. Attene G, Laffaye G, Chaouachi A, Pizzolato F, Migliaccio GM, Padulo J. Repeated sprint ability in young basketball players: one vs. two changes of direction (Part 2). Journal of sports sciences. 2015;33(15):1553-63.

20. Borg GA. Psychophysical bases of perceived exertion. Medicine and science in sports and exercise. 1982;14(5):377-81.

21. Foster C. Monitoring training in athletes with reference to overtraining syndrome. Medicine and science in sports and exercise. 1998;30(7):1164-8.

22. Castagna C, Impellizzeri F, Cecchini E, Rampinini E, Alvarez JC. Effects of intermittent-endurance fitness on match performance in young male soccer players. Journal of strength and conditioning research. 2009;23(7):19549.

23. Cohen J. Eta-Squared and Partial Eta-Squared in Fixed Factor Anova Designs. Educational and Psychological Measurement. 1973;33(1):107-12.

24. Meckel Y, Ismaeel A, Eliakim A. The effect of the Ramadan fast on physical performance and dietary habits in adolescent soccer players. European Journal of Applied Physiology. 2008;102(6):651-7.

25. Suwaidi A, Bener A, Gehani A, Behair S, Mohanadi D, Salam A, et al. Does the circadian pattern for acute cardiac events presentation vary with fasting? Journal of Postgraduate Medicine. 2006;52(1):30-3.

26. Stokholm KH, Breum L, Astrup A. Cardiac contractility, central haemodynamics and blood pressure regulation during semistarvation. Clinical Physiology. 1991;11(6):513-23.

27. Fall A, Sarr M, Mandengue S-H, Badji L, Samb A, Gueye L, et al. Effects of prolonged water and food restriction (ramadan) on performance and cardiovascular responses during incremental exercise in tropical climate. Science Et Sports. 2007;22(1):50-3. 